\section{Parametrization of intensive global climate change indicators on a level of sovereign states and governments - ADDENDUM}

doi: https://doi.org/10.1557/mre.2019.8

The following footnote should be included in this article [1]:

This paper was commissioned and accepted for publication by David Cahen, who served as Editor-in-Chief of this journal from 2014-2018.
Micha Tomkiewicz

REFERENCE:

1. Tomkiewicz M. (2019). Parametrization of intensive global climate change indicators on a level of sovereign states and governments. MRS Energy \& Sustainability, 6, E7. Cambridge University Press. 